The Astrophysical JouRnAL, 522:973-982, 1999 September 10

(c) 1999. The American Astronomical Society. All rights reserved. Printed in U.S.A.

\title{
DIRECT MEASUREMENT OF A SECULAR PULSATION PERIOD CHANGE IN THE PULSATING HOT PRE-WHITE DWARF PG 1159-035
}

\author{
J. E. S. Costa And S. O. KePler \\ Instituto de Física da Universidade Federal do Rio Grande do Sul, 91501-970 Porto Alegre, RS Brazil; edu@if.ufrgs.br, kepler@if.ufrgs.br \\ AND \\ D. E. WINGET \\ Department of Astronomy and McDonald Observatory, University of Texas, Austin, TX 78712-1085; dew@astro.as.utexas.edu \\ Received 1998 January 26; accepted 1999 April 20
}

\begin{abstract}
GW Vir (PG 1159-035) is the prototype of the class of multiperiodic, nonradially pulsating hot white dwarfs, and shows a strong pulsation mode at $516 \mathrm{~s}$. All measurements to date of the secular variation of the $516 \mathrm{~s}$ pulsation quote as best value $\dot{P}=(-2.49 \pm 0.06) \times 10^{-11} \mathrm{~s} \mathrm{~s}^{-1}$. The original measurement gave two best solutions, and a $\chi^{2}$ analysis indicated that the quoted value was preferred at the level of 0.97 probability. On other hand, the best-developed models for planetary nebula nuclei (PNNs), using models from the asymptotic giant branch as starting points and simulating the observed mass loss, provide positive values for any model with $\log \left(L / L_{\odot}\right) \lesssim 3$, as PG $1159-035$. This conflict between the measurement and the theoretical models has been a challenge to stellar evolution theory.

Exploiting a much larger data set and computational techniques previously unavailable, we show that the earlier analysis of the data grossly underestimated the true uncertainties due to interferences between frequencies. Using new data along with the old, and more accurate statistical methods, we calculated the secular period change of the $516 \mathrm{~s}$ pulsation, and obtained a positive value: $\dot{P}=$ $(+13.07 \pm 0.03) \times 10^{-11} \mathrm{~s} \mathrm{~s}^{-1}$. We show that three additional methods yield the same solution. This new value was the second best of the original possible solutions; it was eliminated on the basis of statistical arguments that we show to be invalid. It is an order of magnitude larger than the theoretical predictions.

Additionally, from rotational splitting analysis, we were able to estimate, for the first time, a limit to the secular variation of the rotational period $\dot{P}_{\text {rot }}=(-1.0 \pm 3.5) \times 10^{-11} \mathrm{~s} \mathrm{~s}^{-1}$, leading to a contraction timescale upper limit of $\left|\tau_{R}^{-1}\right|=|\dot{R} / R|<48 \times 10^{-11} \mathrm{~s}^{-1}$ with $99.5 \%$ probability.

Subject headings: stars: evolution - stars: individual (PG 1159-035) — stars: oscillations white dwarfs
\end{abstract}

\section{INTRODUCTION}

The pre-white dwarf star PG 1159-035 was first identified on the PG Survey - a survey for objects with ultraviolet excess (Green 1977). Its variability was discovered by McGraw et al. (1979), who showed that PG 1159-035 was a multiperiodic variable star with at least two pulsation periods simultaneously present in its light curve. Later, other stars were identified with similar photometric and spectroscopic characteristics, defining the spectral class of PG 1159 stars, and, for the variable ones, the GW Vir stars or DOV class, in the nomenclature established by Sion et al. (1983). The Kiel group (Werner 1995; Dreizler 1998) has shown that the best-fitting model for PG $1159-035$ has $T_{\text {eff }}=140,000 \mathrm{~K}$ and $\log g=7.0$.

The presence of $\mathrm{He}$ II absorption lines in its spectrum suggested a very high surface temperature, placing the star on the upper left of the white dwarf sequence on the H-R diagram. The high luminosity and temperature led McGraw et al. (1979) to suggest that PG 1159-035 should be evolving rapidly, causing the pulsation periods to change in response to the changes in the internal structure of the star; they pointed out that the period change should be large enough to be measurable within a few years.

In 1983, Winget, Hansen, \& Van Horn (1983. hereafter WHVH) published a preliminary study for the theoretical estimates of the timescale of the period change, $|P / \dot{P}|$, where $P$ is the pulsation period and $\dot{P}$ is its secular change.
Their main goal was to explore the competing effects of radius and temperature change in determining the secular frequency evolution of nonradial $g$-modes in white dwarf and pre-white dwarf stars. Their estimates, based on crude gravitationally contracting polytropes used as input to their evolutionary code, indicated that the timescale for period change should be of the order of $10^{6}$ years. This timescale implied that the period variation would be measurable in one to three observation seasons, depending on the complexity of the light curve, using the technique of the $(O-C)$ diagram employed by Kepler et al. (1982) to determine the period changes of $\mathrm{ZZ}$ Ceti variable stars. They foresaw a possible negative value for $\dot{P}$ for PG 1159-035, taking into account the large uncertainties of the parameters used in their calculations.

Observations obtained from 1979 to 1984 allowed the identification of eight separate period groups. For the strongest pulsation mode at $516 \mathrm{~s}$, Winget et al. (1985) confirmed its stability and estimated its secular change, $P$. The technique used to estimate $\dot{P}$ was the classical $(O-C)$ diagram, which provides multiple solutions, due to possible uncertainties of an integer number of pulsation cycles from one observational season to the next. They analyzed nine possible solutions, obtained two viable solutions $(\dot{P}=$ $-1.21 \times 10^{-11} \mathrm{~s} \mathrm{~s}^{-1}$ and $\dot{P}=+6.61 \times 10^{-11} \mathrm{~s} \mathrm{~s}^{-1}$ ). The only available method which allowed them to choose between the two solutions was based on $\chi^{2}$ tests, using as 
uncertainties 10 times those derived by single frequency fits. The best fit was obtained with $\dot{P}=(-1.21 \pm 0.06) \times 10^{-11}$ $\mathrm{s} \mathrm{s}^{-1}$. The negative sign agreed with the theoretical estimates of WHVH, but not with the more physically selfconsistent calculations of Kawaler (1986) and Kawaler, Hansen, \& Winget (1985a), which used evolutionary modes as input.

Winget \& Kepler (1988) recalculated $\dot{P}$ by joining the 1979-1984 photometric data to runs obtained in 1985 and 1987 , but now used a new definition for $\dot{P}$ that corrects the previous one by a factor of 2 . This factor of 2 comes from the recognition that $\dot{P}$ is a second derivative of the phase (see Kepler et al. 1991 for more explanations about the definition of $\dot{P})$. For the $1979-1984$ data $\dot{P}=(-2.50$ $\pm 0.12) \times 10^{-11} \mathrm{~s} \mathrm{~s}^{-1}$. The new result obtained with the $(O-C)$ diagram technique for the 1979-1987 data was $\dot{P}=$ $(-2.36 \pm 0.40) \times 10^{-11} \mathrm{~s} \mathrm{~s}^{-1}$. Using this value as an initial guess, they also calculated $\dot{P}$ with a nonlinear least-squares (NLS) fitting, and the result was $\dot{P}=(-2.44$ $\pm 0.06) \times 10^{-11} \mathrm{~s} \mathrm{~s}^{-1}$. The uncertainty quoted is the one for the NLS fitting, which underestimates the actual uncertainty because it assumes all data are uncorrelated, and this is not the case for multiperiodic data.

In 1989 Winget and his collaborators (Winget et al. 1991) obtained 264.1 hours of contemporaneous observations of PG 1159-035 with the Whole Earth Telescope (Nather et al. 1990. Winget et al. (1991) used the data to estimate the stellar mass and a host of other structural information about the star, and obtain another estimate of $\dot{P}$, using the ephemeris from the 1985 fitting. The value then obtained for $\dot{P}$ with the classical method of $(O-C)$ diagram was $\dot{P}=(-2.49 \pm 0.06) \times 10^{-11} \mathrm{~s} \mathrm{~s}^{-1}$, consistent with the earlier results. The 1989 light curve of PG 1159-035 provided a power spectrum with high enough resolution to identify 122 individual frequencies (or periods), with 101 of them corresponding to specific, quantized pulsation modes. The dominant period of $\approx 516 \mathrm{~s}$ appears as the prograde $(m=+1)$ component of a triplet $(\ell=1)$. The rotation period of PG $1159-035$ was found to be $P_{\text {rot }}=+1.38$ \pm 0.01 days. The derived mass was $M=0.586 \pm 0.003 M_{\odot}$.

In $\S 3$ we describe the methods used to compute our new measurements of $\dot{P}$ and present the results. The comparison of these results with the new theoretical models is made in the following section. Section 5 contains a preliminary derivation of the contraction timescale of PG 1159-035 from the rotational splitting analysis. Finally, in $\S 6$, we summarize and discuss our results in the light of stellar evolution theory.

\section{THE NEGATIVE $\dot{P}$ PROBLEM}

The measurements to date of the secular change of the dominant $516 \mathrm{~s}$ period were negative. These results were in agreement with the first theoretical estimates done by WHVH, who used evolutionary calculations with the best treatment available at that time for the interior, envelope, equation of state, and opacities. However, those calculations used polytropes for starting models. This neglected the thermal history of the star from prior evolution. They reached the neighborhood of PG 1159-035 in the H-R diagram by gravitational contraction and heating through a curve with a roughly constant luminosity, at the position of the extended horizontal branch (EHB) (Winget et al. 1991).

Kawaler et al. (1985a) and Kawaler (1986) recalculated the timescale using initial models that were the hot cores of evolutionary asymptotic giant branch (AGB) stars. The envelopes were artificially removed to simulate mass loss and reproduce models of planetary nebula nuclei (PNNs). They found that $\dot{P}$ for the 516 s pulsation period was always positive for PNN models with $\log \left(L / L_{\odot}\right) \lesssim 3$. This result held for any of their models evolved from the AGB; the results were insensitive to the total stellar mass, the surface layer mass and its chemical composition, nuclear shell burning, and core composition. Thus, all these models disagreed with the measured value of $\dot{P}$, prompting the exploration of a number of possible explanations.

Kawaler et al. (1985a) tried to solve this dilemma: they noted that if the $516 \mathrm{~s}$ pulsation mode were an $\ell=2$ or $\ell=3$ mode with $m=\ell$, then $\dot{P}$ might be dominated by the spin-up associated with evolutionary contraction. The rotation period had to be shorter than $\approx 3000 \mathrm{~s}$ to make a sign inversion of $\dot{P}$ for their post-PNN models possible. The rotation period measured by Winget et al. (1991) from the 1989 data is 1.38 days, and the $516 \mathrm{~s}$ is an $\ell=1$ mode, so this possibility had to be discarded.

Winget et al. (1991) pointed out that the only obvious alternatives for the negative $\dot{P}$ problem was if the observed $\dot{P}$ value did not measure the actual cooling rate, and the good agreement between the observed timescale and the cooling theory prediction was only a coincidence. Alternatively, the progenitor star of PG $1159-035$ could be substantially different from the post-planetary nebula models of Kawaler; perhaps it evolved along the EHB, as assumed by the WHVH models.

Kawaler \& Bradley (1994) developed evolutionary models where the negative $\dot{P}$ appears because of "mode trapping." As established by Kawaler (1986) and subsequent investigations, it is the competing effects of contraction and cooling in the region of period formation that dominate the secular period change. Kawaler \& Bradley were able to show that, for some models, mode trapping in the (relatively) less degenerate envelope had rates of period change dominated by contraction rather than cooling. Hence these trapped modes had negative rates of period change. They showed that if this were the real reason for the negative $\dot{P}$ of the $516 \mathrm{~s}$ period, then "nontrapped" pulsation modes, like the $495 \mathrm{~s}$ and $451 \mathrm{~s}$ ones, should show positive secular period variation. On the other hand, the $539 \mathrm{~s}$ mode, which was also trapped on their models, might also show a negative $\dot{P}$. Costa, Kepler, \&Winget (1995) used the $(O-C)$ algorithm developed by O'Donoghue (1994) to estimate the secular variation rate of the $539 \mathrm{~s}$ period. The data included the $\approx 345$ hours of photometric data obtained in 1993, again obtained with the WET. The result for this first measurement was $\dot{P}_{539}=(-0.82 \pm 0.04)$ $\times 10^{-11} \mathrm{~s} \mathrm{~s}^{-1}$, consistent with the mode- trapping hypothesis, but they did not study the nontrapped modes.

\section{NEW MEASUREMENT OF $\dot{P}$}

The importance of understanding this rapid evolutionary phase demands a reinvestigation of the rate of period change with new numerical analysis techniques and the new extensive data sets. To recalculate the secular variation of the $516 \mathrm{~s}$ period, we use four methods. We call the first one the direct method (DM), and discuss it in $\S 3.1$. The second method is based on the maximum likelihood estimation and is discussed in $\S 3.2$. The third method is a variant of the $(O-C)$ algorithm proposed by O'Donoghue (1994), which we describe in $\S 3.3$, pointing out the important factors that 
must be taken into account on any measurement of rates of period change. Finally, in $\S 3.4$ we discuss the nonlinear least-squares (NLS) method.

\subsection{The Direct Method}

Because the value of $\dot{P}$ for the $516 \mathrm{~s}$ period is of order $10^{-11} \mathrm{~s} \mathrm{~s}^{-1}$, in 10 years (1983-1993) PG $1159-035$ should have a period change $|\Delta P|$ of order $10^{-3} \mathrm{~s}$. A change of this size can be measured directly from the power spectra of the annual time series, if the periods $\left\{\hat{P}_{i}\right\}_{i=1, \ldots, N}$ have been determined with enough precision, i.e., if the standard deviations of the periods, $\left\{\sigma_{P_{i}}\right\}_{i=1, \ldots, N}$, are much smaller than the total period variation $\left(\sigma_{P_{i}} \ll|\Delta P|\right)$.

\subsubsection{Phase Dispersion Minimization}

One way to calculate the standard deviations $\sigma_{P_{i}}$ is through the method of phase dispersion minimization (PDM) (Stellingwerf 1978). To use this method, it is necessary to segment the total time series of each year into a set of individual parts and calculate the phase of a sinusoidal signal of period $P_{i}$. This segmentation reduces the precision of the phase estimate. Also, beating between the components of the multiplets introduces systematic (nonrandom) biases in the measured phases, so the formal standard deviations of the phases, $\sigma_{\phi_{i}}$, derived from the least squares method, underestimate the true values (Winget et al. 1985). This occurs, at least in part, as a result of correlation between the sinusoidal components present on the data (Schwarzenberg-Czerny 1989). On the other hand, the $\sigma_{P_{i}}$ derived from the PDM method tends to overestimate the actual values.

\subsubsection{Monte Carlo Simulations}

We derive more realistic values for $\left\{\sigma_{P_{i}}\right\}$ using a method based on Monte Carlo simulations (MCSs) (Costa 1996; see Press et al. 1986 for a general discussion of Monte Carlo techniques). From Fourier analysis of the photometric data, we derive estimates for the parameters - periods, amplitudes, and phases - of the detected pulsation modes. For each MCS we vary the values of all the parameters within predefined ranges. So, for each MCS we have a new set of parameters with actual values known, and an artificial light curve is constructed. Then Fourier analysis is applied to derive estimates for the parameter values. The estimated values are compared with the true (known) values. The differences are the actual errors. Repeating his process many times, we obtain an empirical error distribution for each one of the parameters. The standard deviation of the error distribution of a given parameter is the standard deviation for the estimated value of that parameter.

The MCS method also allows us to test the performance of the least-squares or any other method on the determination of the standard deviations of amplitudes and phases $\left(\sigma_{A}\right.$ and $\left.\sigma_{\phi}\right)$. To illustrate this, we calculate 1000 simulations, using as a model the time series of PG 1159-035 obtained in 1989 (Winget et al. 1991). For each simulation, we calculate how many times the actual error of an estimate of amplitude, $\epsilon_{A}$, or of phase, $\epsilon_{\phi}$, was greater than the standard deviation derived from the least-squares process, $\sigma_{A}^{\mathrm{LS}}$ or $\sigma_{\phi}^{\mathrm{LS}}$. The histograms in Figure 1 show the number of occurrences of values of $(a) \epsilon_{A} / \sigma_{A}^{\mathrm{LS}}$ and $(b) \epsilon_{\phi} / \sigma_{\phi}^{\mathrm{LS}}$ within certain ranges. For a Gaussian distribution, the maximum expected value for $\left|\epsilon_{A} / \sigma_{A}^{\mathrm{LS}}\right|$ or $\left|\epsilon_{\phi} / \sigma_{\phi}^{\mathrm{LS}}\right|$ for 1000 simulations is 3.48 . The histograms clearly show that both rates greatly exceed the limit of 3.48 , justifying also the use of the MCS method to calculate $\sigma_{\phi}$.

All least-squares procedures calculate $\sigma_{x}^{2}=\sum\left(x_{\mathrm{obs}}\right.$ $\left.-x_{\text {calc }}\right) /\left(N_{\text {unc }}-k\right)$, where $N_{\text {unc }}$ is the number of uncorrelated data points and $k$ is the number of parameters in the fit. The problem is to estimate the number of uncorrelated points. Most of the calculations assume all the data points are uncorrelated, which is not true for multiperiodic data. As a minimum, we have to subtract from the real number of points, $N_{\text {tot }}$, the number of implicit parameters, 2 for each periodicity known.

\subsubsection{The Sinusoidal Subtraction Technique}

It is possible to reduce the errors in the estimates of a given period (and also of phase and amplitude) by subtracting from the original time series data - the light curve - the
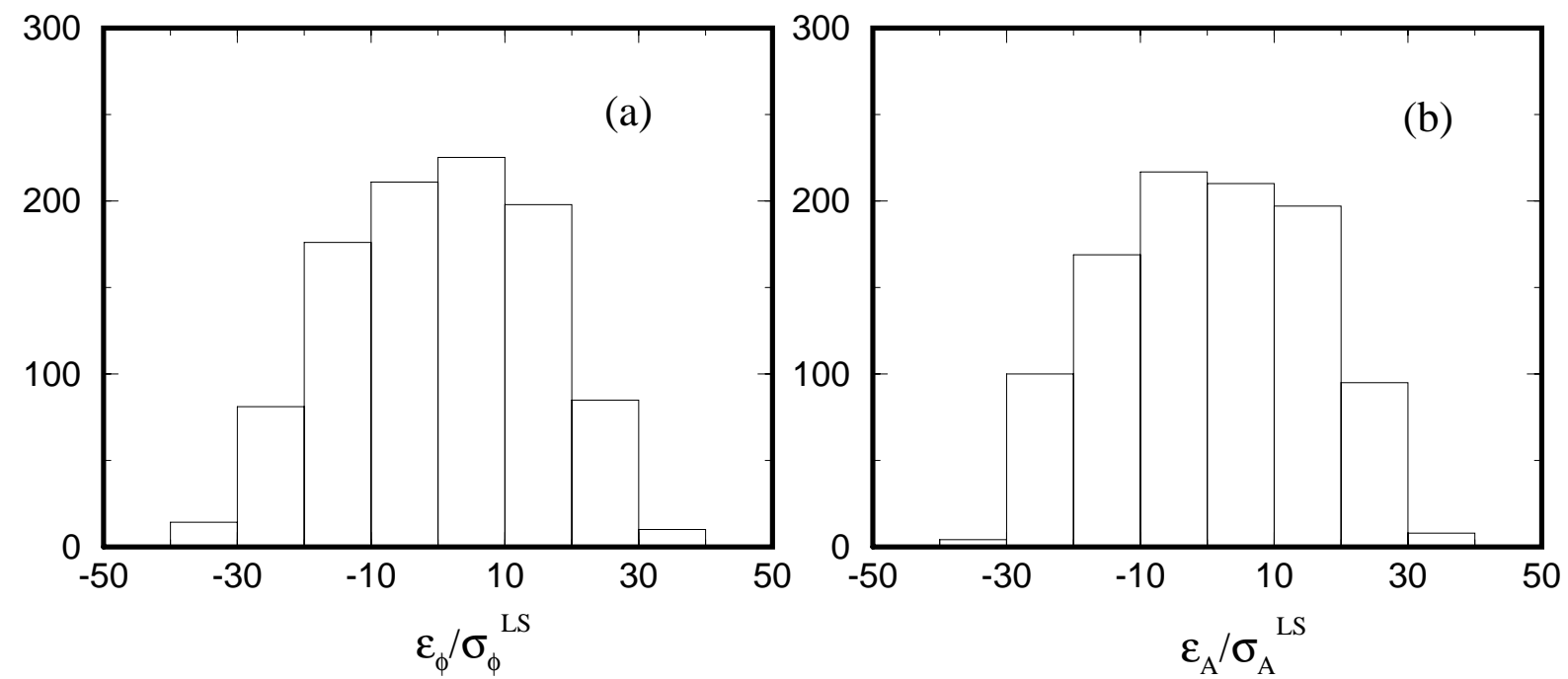

FIG. 1.-For each simulation, we calculated how many times the actual error of an estimate of amplitude, $\epsilon_{A}$, or phase, $\epsilon_{\phi}$, was greater than the standard deviation derived from the least-squares process, $\sigma_{A}^{\mathrm{LS}}$ or $\sigma_{\phi}^{\mathrm{LS}}$. The histograms above show the number of occurrences of values of $(a) \epsilon_{A} / \sigma_{A}^{\mathrm{LS}}$ and $(b) \epsilon_{\phi} / \sigma_{\phi}^{\mathrm{LS}}$ within certain ranges. For a Gaussian distribution, the maximum expected value for $\left|\epsilon_{A} / \sigma_{A}^{\mathrm{LS}}\right|$ or $\left|\epsilon_{\phi} / \sigma_{\phi}^{\mathrm{LS}}\right|$ for 1000 simulations is 3.48 . The histograms clearly show that both rates surmount the limit of 3.48. 
largest possible number of other known periods that are present in the data set. This technique is usually called "prewhitening." Figure 2 shows the error distributions for the estimates of $(a)$ the period, $(b)$ the phase, and $(c)$ the amplitude of the $516 \mathrm{~s}$ component of the 1989 data set. The histograms on top show the error distributions before the subtraction, and the lower histograms show the error distributions after the subtraction of the other 121 resolved frequencies identified in the 1989 power spectrum of PG 1159-035 (Winget et al. 1991).

\subsubsection{Application to PG 1159-035}

For the direct method (DM), we use all the photometric data obtained during 1983, 1985, 1989, and 1993. The data sets of 1979, 1980, 1981, and 1984 are too small to provide accurate periods. We determine the periods from power spectra calculated with the DFT algorithm (Deeming 1975) after the subtraction of the largest possible number of sinusoidal curves from the original data sets. It was significant to subtract 121 sinusoidal curves from the 1989 time series, 14 from 1993, and 7 for 1983 and 1985. The $\sigma_{P_{i}}$ were calculated with the MCS method. Table 1 shows our results, and Figure 3 shows the period of the $516 \mathrm{~s}$ mode over time. Assuming a linear period variation,

$$
P(t)=P_{r}+(\Delta P / \Delta t)\left(t-t_{r}\right),
$$

where $t_{r}$ is a reference date and $P_{r}=P\left(t_{r}\right)$, we obtain

$$
\begin{gathered}
P_{r}=516.0271 \pm 0.0008 \mathrm{~s}, \\
\dot{P}=\Delta P / \Delta t=(+13.0 \pm 2.6) \times 10^{-11} \mathrm{~s} \mathrm{~s}^{-1},
\end{gathered}
$$

with $t_{r}=2,445,394.86339$ BJDD (1983). The measured $\Delta P / \Delta t$ for the $516 \mathrm{~s}$ period is therefore positive, and an order of magnitude greater than the previous measurement based on $(O-C)$ methods. The pulsational timescale $\tau_{P}$ is

$$
\tau_{P}=P / \dot{P}=(1.26 \pm 0.25) \times 10^{5} \mathrm{yr} .
$$

This positive solution for $\dot{P}$ was present in the Winget et al. (1985) calculations, but was discarded as being of much lower probability then the negative solution.

\subsection{Maximum Likelihood Estimation}

Another way to estimate the values $P_{r}$ and $\dot{P}$ that better fit the data of Table $1,\left\{\bar{t}_{i}, \hat{P}_{i}\right\}$, is calculating a function $\wp_{g}\left(P_{r}, \dot{P}\right)$ that gives the density of probability that a given couple $\left(P_{r}, \dot{P}\right)$ fits the data. The function is calculated for each point of a grid of values of $P_{i}$ against $\dot{P}_{j}$ (see Fig. 4), and the point that maximizes the function is the solution. This form of parameter estimation is a maximum likelihood estimation (MLE).

The probability $\wp_{g}(P, \dot{P}) \delta P \delta \dot{P}$ that the actual value of $P$ is in the range $[P-\delta P / 2, P+\delta P / 2]$ and that the actual value of $\dot{P}$ is in the range $[\dot{P}-\delta \dot{P} / 2, \dot{P}+\delta \dot{P} / 2]$ is given by (see Fig. 4):

$$
\begin{aligned}
\wp_{g}(P, \dot{P}) \delta P \delta \dot{P}=\prod_{i=1}^{N}\left\{\frac{1}{\sqrt{2 \pi} \sigma_{P_{i}}}\right. & \exp \left[-\frac{\left(P_{i}-\hat{P}_{i}\right)^{2}}{2 \sigma_{P_{i}}^{2}}\right] \\
\times & \left.\frac{1}{2}\left[\delta P+\left(\bar{t}_{i}-\bar{t}_{r}\right) \delta \dot{P}\right]\right\},
\end{aligned}
$$

with $P_{i}=P+\dot{P}\left(\bar{t}_{i}-\bar{t}_{r}\right)$, where $\bar{t}_{i}$ is the average of the timings of the series $S_{i}=\left\{t_{m}, f\left(t_{m}\right)\right\}_{m=1, \ldots, M_{i}}\left(\bar{t}_{i}=\left\langle t_{m}\right\rangle\right)$ and $P_{i}$ and $\sigma_{P_{i}}$ are the measured period and its standard deviation derived from the time series $S_{i}$. Figure 5 shows a plot of the probability density function, $\wp_{g}(P, \dot{P})$, for the four points of Table 1 . The surface is a Gaussian, and its maximum occurs at

$$
\begin{gathered}
P=P_{r}=516.0264 \pm 0.0004 \mathrm{~s}, \\
\dot{P}=(+13.26 \pm 0.52) \times 10^{-11} \mathrm{~s} \mathrm{~s}^{-1} .
\end{gathered}
$$

The pulsational timescale is:

$$
\tau_{P}=(1.23 \pm 0.05) \times 10^{5} \mathrm{yr} .
$$

Note that each point $\left(P_{i}, \dot{P}_{j}\right)$ over the grid defines a straight line, $P(t)=P_{i}+\dot{P}_{j} t$, on the graphic of Figure 3 .

\subsection{The Modified $(O-C)$ Algorithm}

The $(O-C)$ diagram may be defined as a method of phase dispersion minimization of second order with respect to the number of cycles, $E$. We use it to refine the results obtained with the direct method. Usually the phases of a periodic signal of period $P$ are defined as the timings of pulse maxima. If we assume that the period is changing linearly with time (eq. [1]), then the calculated phase (or time of maxima), $C_{i}$, for the time series $S_{i}$ is given by

$$
C_{i}=T_{r}+P_{r} E_{i}+\frac{1}{2} P_{r} \dot{P} E_{i}^{2},
$$

where $T_{r}$ is a time of maximum adopted as the reference date, and $P_{r}$ is the actual period at the date $T_{r}$ (Kepler et al. 1991).

If $O_{i}$ is the observed phase, the dispersion of the observed phases with respect to equation (9) is given by

$$
\chi^{2}\left(P_{r}, \dot{P}\right)=\sum_{i=1}^{N} \frac{\left(O_{i}-C_{i}\right)^{2}}{\sigma_{i}^{2}},
$$

where $\sigma_{i}^{2}=\sigma_{O_{i}}^{2}+\sigma_{C_{i}}^{2}$. The number of cycles $E_{i}$ is calculated from the positive root of equation (9) as $C_{i}=O_{i}$ and $T_{r}=$ $O_{r}$. The $(O-C)$ diagram method consists in finding the values of $T_{r}, P_{r}$, and $\dot{P}$ that minimize equation (10). One way is to calculate $\chi^{2}\left(P_{j}, \dot{P}_{k}\right)$ for all points $\left(P_{j}, \dot{P}_{k}\right)$ over a grid (O'Donoghue 1994; de Jager, Meintjes, \& O'Donoghue 1994). For a given value of $\chi^{2}$, the probability $Q$ that a value of $\chi^{2}$ as poor as the value of equation (10) should occur by chance is given by the incomplete gamma function (Press et al. 1986):

$$
Q\left(P_{j}, \dot{P}_{k}\right)=\Gamma\left(\frac{\chi^{2}}{2}, \frac{N}{2}\right) .
$$

This makes $Q$ an estimate of the goodness of fit of the data. Typically, if $Q$ is larger than 0.1, the goodness of fit is believable. If it is larger than 0.001 , then the fit may be acceptable if the uncertainties do not obey a normal distribution or have been moderately underestimated. If $Q$ is less than 0.001, then the results and/or estimation procedure are doubtful (Press et al. 1986).

Note that the observed phase $O_{i}$ is a function of the period $P_{i}$ via $O_{i}=\phi_{\mathrm{LS}}\left(S_{i}, P_{i}\right)$. Therefore, a small error in the 

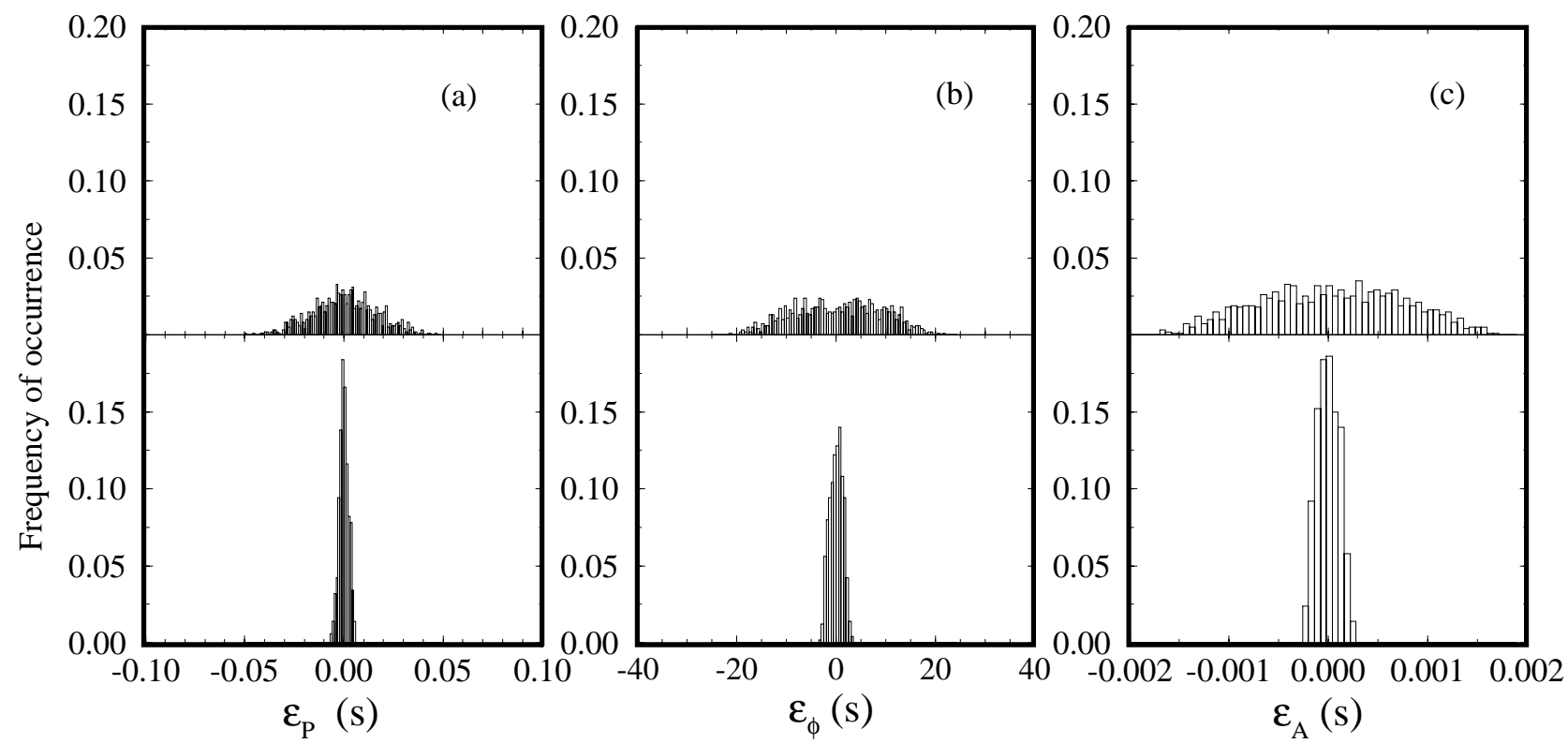

Fig. 2.-Histograms show the error distributions in the estimates of $(a)$ period, $(b)$ phase, and $(c)$ amplitude of the $516 \mathrm{~s}$ component before (top) and after (bottom) the sinusoidal subtractions.

period, $\delta P_{i}$, causes an error in the phase $\delta O_{i}$, where $\delta O_{i} \simeq$ $\left(d \phi_{i} / d P\right) \delta P$. In other words, uncertainties in period complicate interpretation of uncertainties in phase. This problem may be aggravated if $\left|d \phi_{i} / d P\right|$ is large. Figure 6 shows the

TABLE 1

\begin{tabular}{cccc}
\multicolumn{5}{c}{ MEASUREMENTS OF THE PERIODS } \\
\hline \hline Year & $\begin{array}{c}\text { Date }\left(T_{\max }\right) \\
(\text { BJDD })\end{array}$ & $\begin{array}{c}\text { Period } \\
(\mathrm{s})\end{array}$ & $\begin{array}{c}\sigma_{P(\text { MCS })} \\
(\mathrm{s})\end{array}$ \\
\hline $1983 \ldots \ldots$ & $2,445,394.86339$ & 516.0264 & $0.3 \times 10^{-3}$ \\
$1985 \ldots \ldots$ & $2,446,179.97375$ & 516.0372 & $3.9 \times 10^{-3}$ \\
$1989 \ldots \ldots$ & $2,447,592.55883$ & 516.0514 & $2.8 \times 10^{-3}$ \\
$1993 \ldots \ldots$ & $2,449,073.82019$ & 516.0691 & $1.5 \times 10^{-3}$ \\
\hline
\end{tabular}

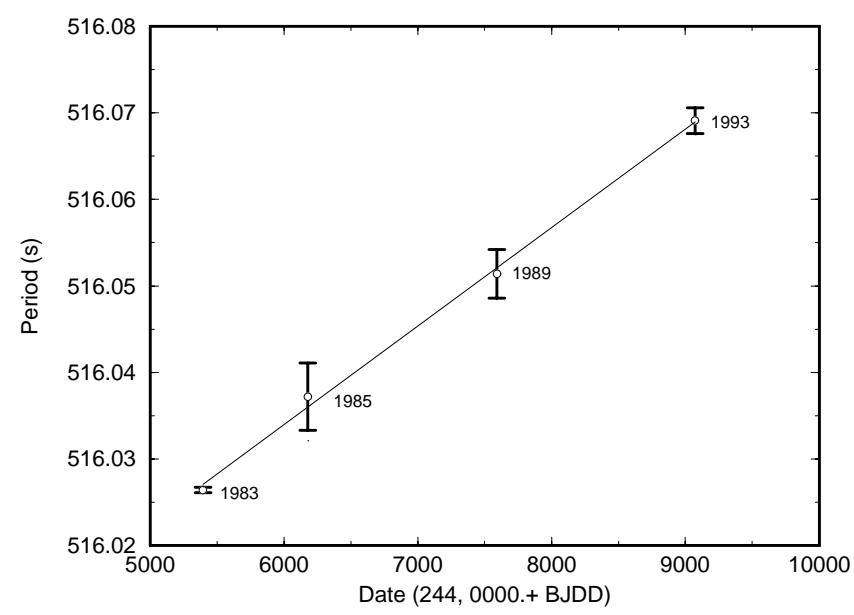

FIG. 3.-Variations of the $516 \mathrm{~s}$ period of PG $1159-035$. The period variation is well fitted by a straight line with a slope of $(+13.0 \pm 2.6) \times 10^{-11} \mathrm{~s} \mathrm{~s}^{-1}$. functions $\phi\left(S_{i}, P\right)$ for PG 1159-035 data from the years of 1980-1993 between 516.0 and $516.1 \mathrm{~s}$. For small ranges of $P$, the curves $\phi\left(S_{i}, P\right)$ are roughly straight lines and their slopes $\left(d \phi_{i} / d P\right)$ depend on several factors, such as the number of data points in each data set, the length, and the size of the daily gaps. Obviously, the problem is more serious for large slopes, and for small slopes it may be neglected. The MCS method takes into account the effect of the uncertainty on the period determination on the estimates of $\sigma_{\phi}$.

As was seen in $\S 3.2$, the points over the grid do not have the same probability of being true. So it is necessary to

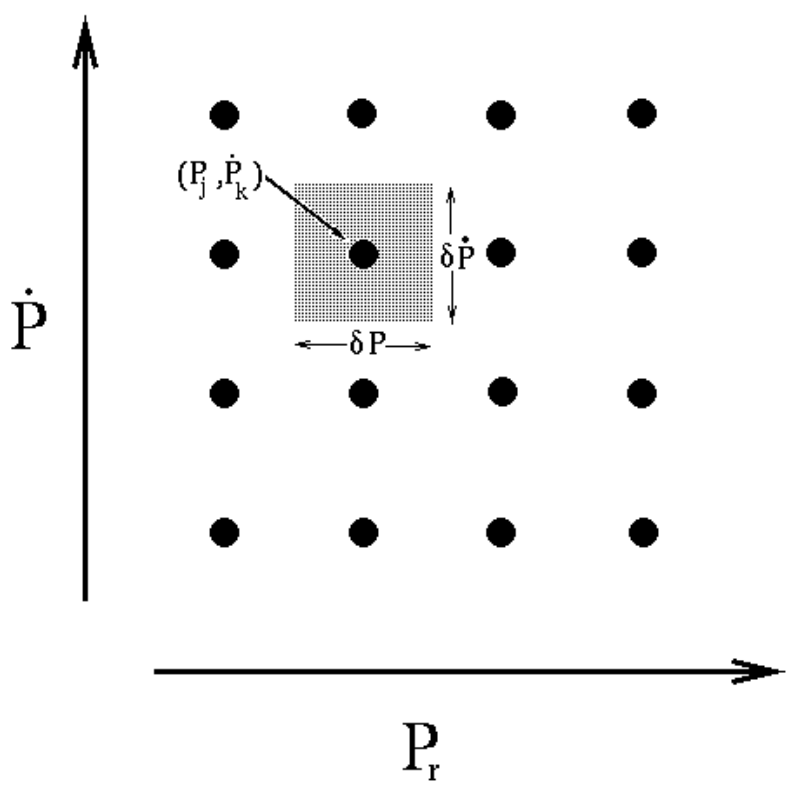

FIG. 4.- Grid of $P_{r}$ vs. $\dot{P}$. The spacing between two consecutive values of $P_{r}$ and $\dot{P}$ are $\delta P$ and $\delta \dot{P}$, respectively. 


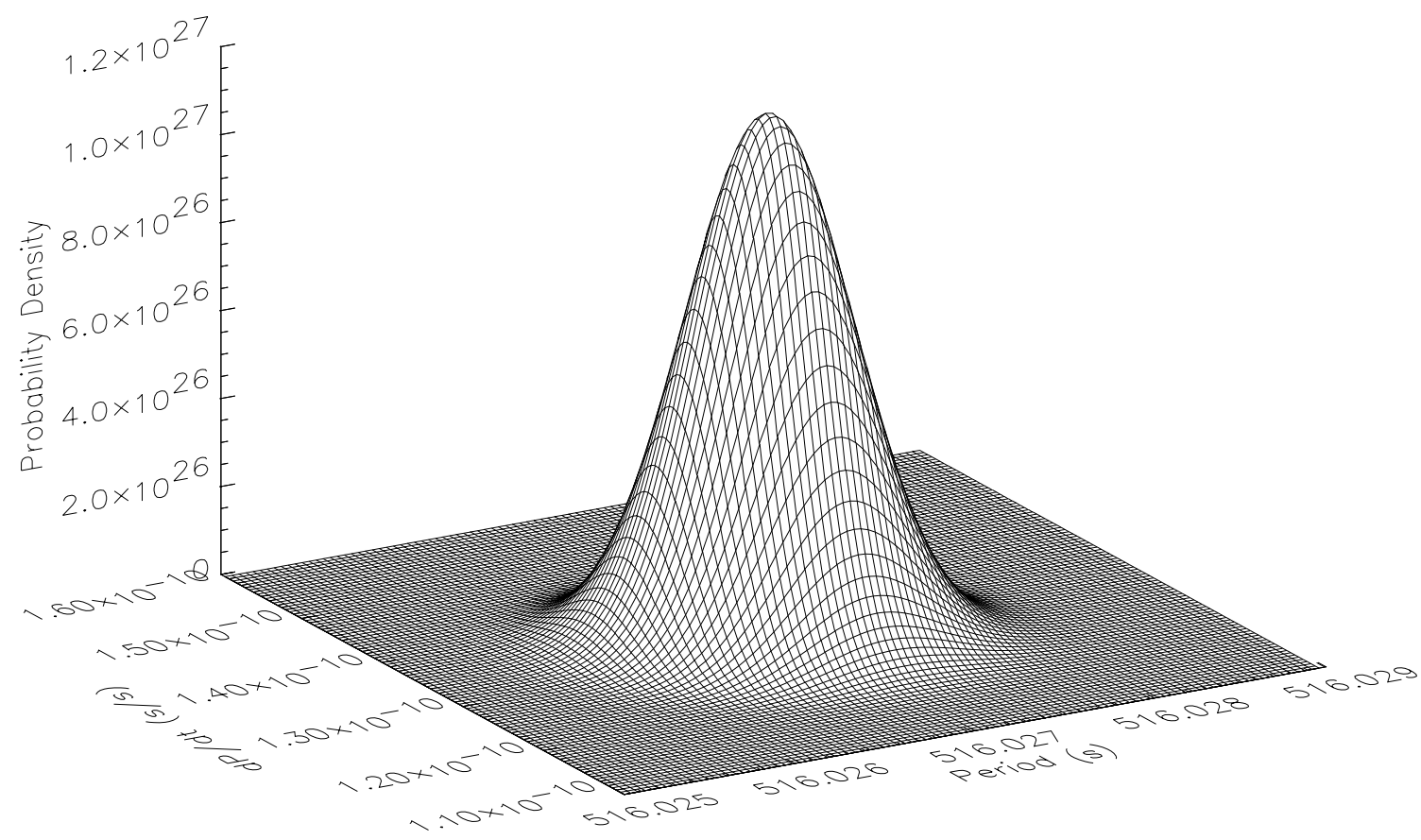

FIG. 5.-Probability density function, $\wp_{g}(P, \dot{P})$

multiply $Q$ from equation (11) by $\wp_{g} \delta P \delta \dot{P}$ from equation (5):

$$
\wp(P, \dot{P}) \delta P \delta \dot{P}=Q(P, \dot{P}) \wp_{g}(P, \dot{P}) \delta P \delta \dot{P} .
$$

The graph of equation (12) is shown in Figure 7. The five best results obtained with this variant of the $(O-C)$ method

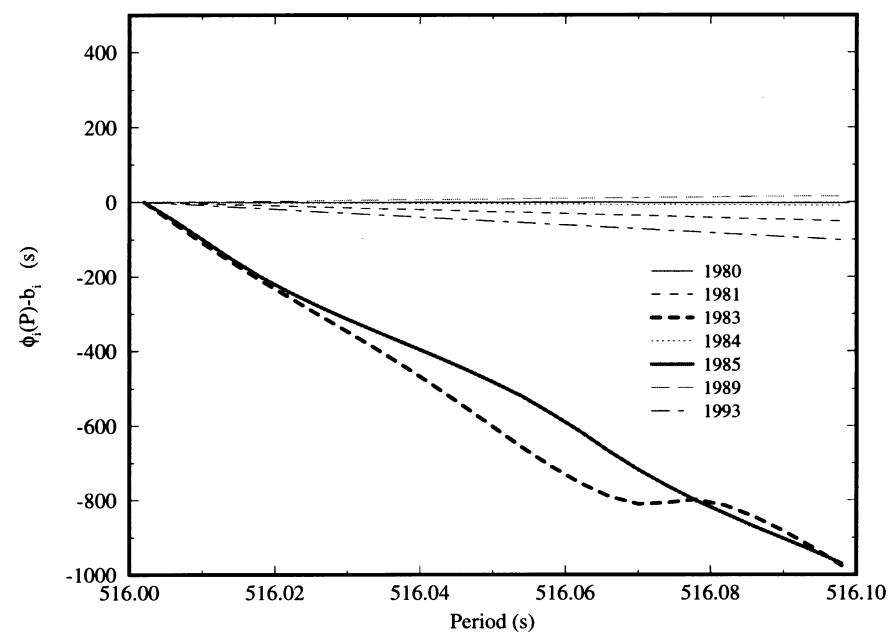

Fig. 6. - Phase as a function of period $P \approx 516 \mathrm{~s}$ for each annual time series $S_{i}, \phi\left(S_{i}, P\right)-b_{i}$. The term $b_{i}$ is chosen so that $\phi\left(S_{i}, P\right)-b_{i}=0$ for $P=516.0$ s. Note that the largest data sets occur in 1983, 1989, and 1993. are shown in Table 2 . All other solutions have $\wp$ less than $10^{20}$. The best solution is

$$
\begin{gathered}
P=P_{r}=516.02679 \pm 0.00007 \mathrm{~s}, \\
\dot{P}=(+13.07 \pm 0.03) \times 10^{-11} \mathrm{~s} \mathrm{~s}^{-1},
\end{gathered}
$$

with a pulsational timescale of

$$
\tau_{P}=\frac{P}{\dot{P}}=(1.25 \pm 0.05) \times 10^{5} \mathrm{yr} .
$$

Figure 8 shows the $(O-C)$ diagram for this result (solid line) and for the result obtained by Winget et al. (1991) (dashed line). The solid points are the average one-cycle alias for each one of the seven epochs involved.

\subsection{Nonlinear Least-Squares Analysis}

The fourth method used to measure the rate of change of the $516 \mathrm{~s}$ pulsation period of PG $1159-035$ was the nonlinear least-squares analysis (NLS), where a model of the form

$$
f\left(t_{i}\right)=A \sin \left\{\left[\omega+\frac{1}{2} \dot{\omega}\left(t-t_{\omega}\right)\right]\left(t-t_{\omega}\right)\right\}
$$

is fitted to the whole original (not prewhitened) data set.

\begin{tabular}{|c|c|c|c|c|c|}
\hline Number & $\begin{array}{l}P \\
(\mathrm{~s})\end{array}$ & $\begin{array}{c}\dot{P} \\
\left(\times 10^{-11} \mathrm{~s} \mathrm{~s}^{-1}\right)\end{array}$ & $Q$ & $\wp_{g}$ & $\wp$ \\
\hline $1 \ldots \ldots$ & 516.02679 & +13.07 & 0.010 & $1.9 \times 10^{28}$ & $2.0 \times 10^{25}$ \\
\hline $2 \ldots \ldots \ldots$ & 516.02722 & +13.86 & 0.224 & $2.0 \times 10^{26}$ & $4.5 \times 10^{24}$ \\
\hline $3 \ldots \ldots \ldots$ & 516.02661 & +14.69 & 0.143 & $1.1 \times 10^{26}$ & $1.6 \times 10^{24}$ \\
\hline $4 \ldots \ldots \ldots$ & 516.02563 & +12.73 & 0.009 & $4.8 \times 10^{26}$ & $4.2 \times 10^{23}$ \\
\hline $5 \ldots \ldots \ldots$ & 516.02740 & +12.21 & 0.014 & $3.8 \times 10^{25}$ & $5.5 \times 10^{22}$ \\
\hline
\end{tabular}
The algorithm searches on the neighborhood of initial values for the values of $A, \omega, \dot{\omega}$, and $t_{\omega}$. The method is very

TABLE 2

Best Results from the Modified $(O-C)$ Algorithm 


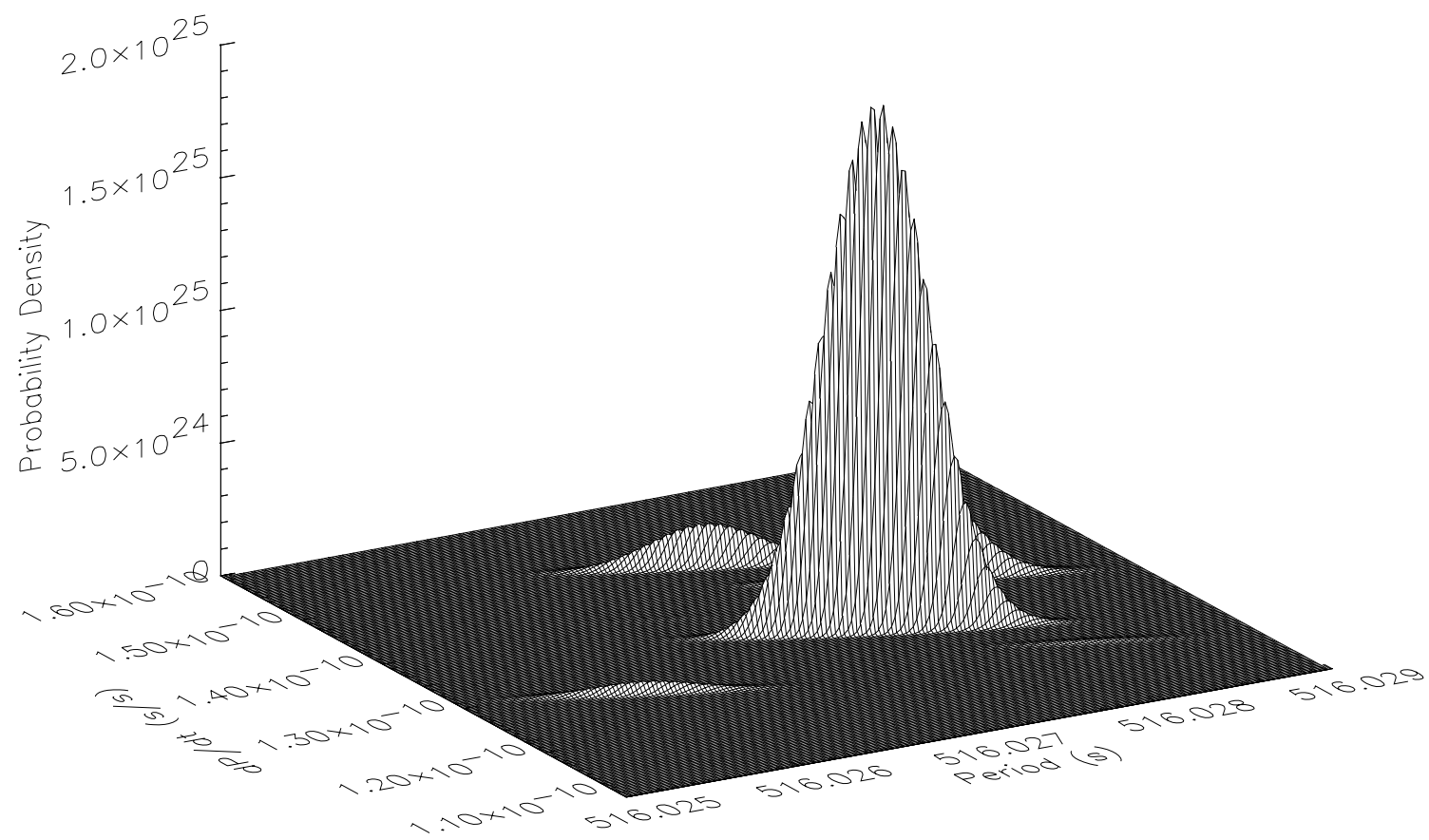

FIG. 7.-Probability density function $\wp(P, \dot{P})$ obtained with the modified $(O-C)$ method. The four best solutions are indicated.

sensitive to initial values, and the computed standard deviations are, in general, much smaller than those for all other three methods. They assume each individual data point is uncorrelated from any other, untrue for multiperiodic data.

We used as initial values the values provided by the $(O-C)$ method, and the obtained results were

$$
\begin{gathered}
T_{0}=2,447,593.341310 \pm 0.000014 \mathrm{BJDD}, \\
P=516.051986 \pm 0.000004 \mathrm{~s} \\
\dot{P}=(+13.298 \pm 0.003) \times 10^{-11} \mathrm{~s} \mathrm{~s}^{-1}
\end{gathered}
$$

The pulsational timescale is:

$$
\tau_{P}=(1.2297 \pm 0.0002) \times 10^{5} \mathrm{yr} .
$$

The quoted uncertainties are the formal (internal) uncer-

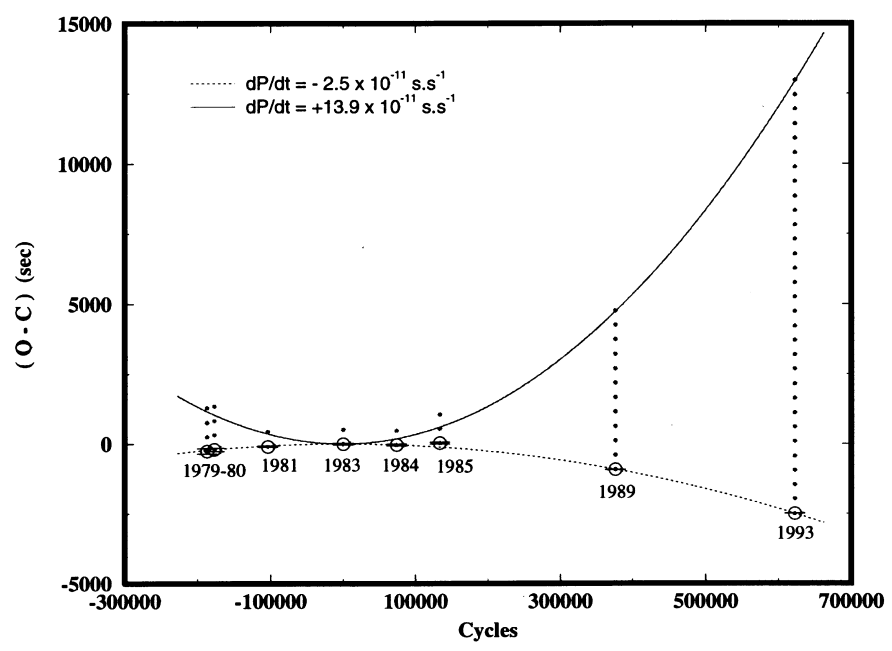

FIG. 8. $-(O-C)$ diagram for the best result (I) and for the solution obtained by Winget et al. (1991) (I). The solid points are the average one-cycle alias for each one of the seven epochs involved. tainties, and are too small for the reasons stated, but are included here to demonstrate the danger they represent.

\section{COMPARISON WITH THE MODELS}

Kawaler \& Bradley (1994) developed a new grid of "quasi-standard" stellar evolutionary models (hereafter KB models) appropriate for PG 1159 stars, and studied the effect of various model parameters on the rate of period change. The parameters of the models were the mass $\left(M_{\star}\right)$, effective temperature $\left(T_{\text {eff }}\right)$, surface helium layer thickness $\left(q_{\mathrm{Y}}\right.$, in stellar masses), and surface composition $\left(T_{\text {surf }}\right)$. Comparison of the KB models with the observed pulsational spectrum of PG $1159-035$ yields a best fit with a mass of $0.59 \pm 0.01 M_{\odot}$, an effective temperature of $\approx 136,000 \mathrm{~K}$, and a He-rich layer of $\sim 0.004 M_{\star}$ with $Y_{\text {surf }} \simeq 0.27$. The nearest model period from $516 \mathrm{~s}$ was $P=519 \mathrm{~s}$ with a rate of change $\dot{P} \simeq 1 \times 10^{-11} \mathrm{~s} \mathrm{~s}^{-1}, 1$ order of magnitude less than our value.

The KB models are very sensitive to stellar mass and effective temperature. The magnitude of $\dot{P} / P$ for a given period generally decreases for increasing stellar mass between 0.50 and $0.80 M_{\odot}$ when the other model parameters are maintained constant, because more massive models have greater ages, hence larger evolutionary timescales. The magnitude of $\dot{P} / P$ also decreases with decreasing $T_{\text {eff }}$, especially for less massive models. Core composition also affects the rate of period change: models with core composition with O-rich mixtures generally have larger $\dot{P} / P$ values than models with $C$-rich mixtures. This is caused by the increased neutrino emission - and faster evolutionary timescales - of the O-rich models. The behavior of $\dot{P} / P$ is also sensitive to the helium surface layer, especially for the trapped modes, because for thinner surface layers the trapping resonance is stronger, resulting in smaller (and possibly negative) $\dot{P} / P$ values (Kawaler \& Bradley 1994 ). Thus, small variations in the parameters may accommodate the large observed $\dot{P}$. 


\section{THE CONTRACTION TIMESCALE}

The frequency splitting observed in the power spectra of nonradial pulsating stars can be caused by two different physical effects: slow rotation and magnetic fields. When the cause is essentially slow rotation, the rotation period may be inferred from the observed frequency splitting as $P_{\text {rot }} \sim 1 / \delta v_{\ell}$, where $\delta v_{\ell}$ is the average difference between two successive frequencies of the same multiplet. The data analysis shows that this is the case of PG 1159-035 (Winget et al. 1991). In this section we demonstrate the use of the rotational splitting analysis to estimate roughly the contraction timescale of a pulsating white dwarf and show preliminary results for PG $1159-035$.

\subsection{Observations of $\Delta P / \Delta t$ in the $516 \mathrm{~s}$ Multiplet}

The $516 \mathrm{~s}$ period appeared in the analysis of the data of 1979-1985 as a singlet (Winget et al. 1985; Winget \& Kepler 1988). In the high-resolution power spectrum obtained with the $264.1 \mathrm{hr}$ of quasi-continuous photometric data of 1989 (Winget et al. 1991), the band of power at $516 \mathrm{~s}$ became a triplet of $\ell=1$ modes, with the previously studied period at $516 \mathrm{~s}$ appearing as the $m=+1$ component. This triplet is also clear in the power spectrum obtained in 1993. The two other members of the triplet, with periods at $\approx 517 \mathrm{~s}(m=0)$ and $\approx 518 \mathrm{~s}(m=-1)$, were of sufficiently low amplitude until 1987 that they had little or no effect on the apparent phase and frequency of the $516 \mathrm{~s}$ band (Winget et al. 1991).

Figure 9 shows the $516 \mathrm{~s}$ triplet in the 1989 (top) and 1993 (bottom) power spectra. As we can clearly see, the three modes of the triplet appear to be stable with respect to pulsation period, but only the $m=+1$ mode is stable with respect to amplitude. Furthermore, Winget et al. (1985, 1991 and Winget \& Kepler 1988) have pointed out that the data show that many of the other pulsation periods, including the $m=0$ and $m=-1$ modes of the $516 \mathrm{~s}$ triplet are unstable over the period of time covered by the observations, which is confirmed by the data from 1993. In Table 3 we present the measurements of the periods of the three components of the triplets. Their standard deviations come from the MCS method. The rates of period variation from 1989 to $1993, \Delta P / \Delta t$, are in the last row. The rates of variation of the periods of $517 \mathrm{~s}$ and $518 \mathrm{~s}$ are of the same order of magnitude as the $516 \mathrm{~s}$ period, but the present errors are too large for $\Delta P / \Delta t$ to be significant.

\subsection{Estimation of the Contraction Timescale}

The rotational splitting of $g$-modes depends on the rotation law. For slow rotation $\left(P_{\text {rot }} \gg P_{\text {puls }}\right)$, the expression for the rotational splitting is given by (Hansen, Cox, \& Van Horn 1977; Kawaler, Winget, \& Hansen 1985b; Winget et al. 1991)

$$
\sigma_{k \ell m}=\sigma_{k \ell}+m\left(1-C-C_{1}\right) \Omega_{\mathrm{rot}}+O\left(\Omega_{\mathrm{rot}}^{2}\right),
$$

where the last term indicates terms of second order in the rotation frequency, $\Omega_{\mathrm{rot}} ; C[=C(k, \ell)]$ is the uniform rota-

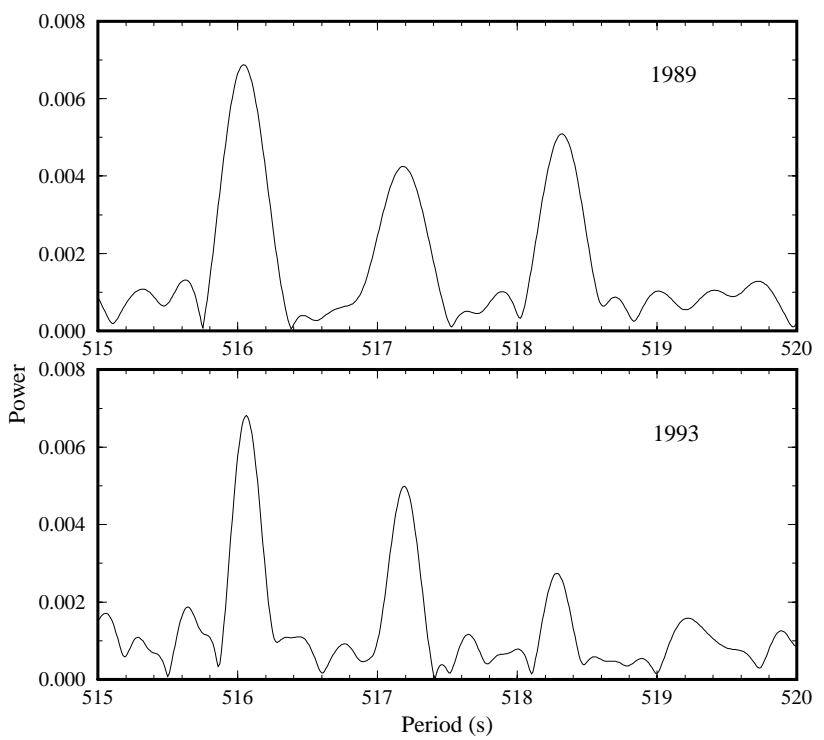

Fig. 9.-The $516 s$ triplet $(\ell=1)$ at the 1989 (top) and 1993 (bottom) power spectra. The $516 \mathrm{~s}$ period is the prograde component $(m=+1)$, the $517 \mathrm{~s}$ period is the central component $(m=0)$, and the $518 \mathrm{~s}$ period is the retrograde component $(m=-1)$.

tion coefficient; $C_{1}\left[=C_{1}(k, \ell,|m|)\right]$ contains the nonuniform rotation effects; $\sigma_{k \ell m}$ is the angular frequency of pulsation with $\sigma_{k \ell}=\sigma_{k \ell 0}(m=0)$. The value of $C_{1}$ depends on the adiabatic pulsation properties, the equilibrium structure of the star, and the rotation law.

If we neglect second-order terms in $\Omega_{\text {rot }}$, differentiate, and transform the frequencies to periods, we obtain (Kawaler et al. 1985b)

$$
\frac{\dot{P}_{m}}{P_{m}^{2}}=\frac{\dot{P}_{0}}{P_{0}^{2}}+m\left(1-C-C_{1}\right) \frac{\dot{P}_{\text {rot }}}{P_{\text {rot }}^{2}}+m\left(\dot{C}+\dot{C}_{1}\right) \frac{1}{P_{\text {rot }}},
$$

where $P_{\text {rot }}$ is the rotational period at the pole. In the asymptotic limit of high radial overtones, i.e., large values of $k$ (Brickhill 1975), the uniform rotation coefficient can be approximated by

$$
C \simeq \frac{1}{\ell(\ell+1)} .
$$

If we assume uniform rotation as a first approximation, then $C_{1}=0$ and $\dot{C}=\dot{C}_{1}=0$. For $\ell=1$, equation (22) becomes

$$
\frac{\dot{P}_{m}}{P_{m}^{2}}=\frac{\dot{P}_{0}}{P_{0}^{2}}+\frac{1}{2} m \frac{\dot{P}_{\text {rot }}}{P_{\text {rot }}^{2}} .
$$

For a star with uniform rotation, the angular momentum is $J \propto M R^{2} \Omega_{\text {rot }}$. If the angular momentum is conserved,

TABLE 3

THE $516 \mathrm{~s}$ TRIPLET

\begin{tabular}{ccccc}
\hline \hline & $\begin{array}{c}P_{1989} \\
(\mathrm{~s})\end{array}$ & $\begin{array}{c}P_{1993} \\
(\mathrm{~s})\end{array}$ & $\Delta P=P_{1993}-P_{1989}$ \\
$(\mathrm{~s})$ & $\begin{array}{c}\dot{P}_{m}=\Delta P / \Delta t \\
\left(\times 10^{-11} \mathrm{~s} \mathrm{~s}^{-1}\right)\end{array}$ \\
\hline$+1 \ldots \ldots$ & $516.0514 \pm 0.0028$ & $516.0691 \pm 0.0015$ & $+0.0177 \pm 0.0032$ & $+13.83 \pm 2.50$ \\
$0 \ldots \ldots$ & $517.1569 \pm 0.0185$ & $517.1863 \pm 0.0231$ & $+0.0294 \pm 0.0296$ & $+22.97 \pm 23.13$ \\
$-1 \ldots \ldots$ & $518.2932 \pm 0.0355$ & $518.2884 \pm 0.0327$ & $-0.0050 \pm 0.0482$ & $-3.91 \pm 37.66$ \\
\hline
\end{tabular}




$$
d J / d t=0 \text {, and }
$$

$$
R^{2} \Omega_{\mathrm{rot}} \dot{M}+2 M R \Omega_{\mathrm{rot}} \dot{R}+M R^{2} \dot{\Omega}_{\mathrm{rot}}=0,
$$

where $\dot{M}, \dot{R}$, and $\dot{\Omega}_{\text {rot }}$ are the temporal derivatives of the mass $(M)$, radius $(R)$, and rotation frequency $\left(\Omega_{\mathrm{rot}}\right)$ of the star. If mass loss in the present stage is negligible $(\dot{M} \simeq 0)$, then equation (25) becomes:

$$
\frac{\dot{R}}{R} \simeq \frac{1}{2} \frac{\dot{P}_{\text {rot }}}{P_{\text {rot }}} .
$$

As the star evolves and cools, it will contract $(\dot{R}<0)$, and therefore the rotational period will decrease $\left(\dot{P}_{\text {rot }}<0\right)$ to conserve the angular momentum. A negative $\dot{P}_{\text {rot }}$, according to equation (24) implies $\dot{P}_{m=1}<\dot{P}_{m=0}$, which is consistent with Table $5\left(\dot{P}_{516}<\dot{P}_{517}\right)$, but this result is not significant. As we have estimated $\dot{P}_{0}$ and $\dot{P}_{m}$, we can use equation (24) to obtain a rough estimate for the secular variation of the rotational period:

$$
\dot{P}_{\text {rot }}=(-1.0 \pm 3.5) \times 10^{-5} \mathrm{~s} \mathrm{~s}^{-1},
$$

i.e., the rotational period changes by an amount of the order of a few minutes per year. Using this result, we can now estimate from equation (26) the timescale for radius change, $\tau_{R}^{-1}=R / \dot{R}:$

$$
\begin{gathered}
\frac{\dot{R}}{R}=\frac{1}{m} P_{\text {rot }}\left(\frac{\dot{P}_{m}}{P_{m}^{2}}-\frac{\dot{P}_{0}}{P_{0}^{2}}\right) \quad(m \neq 0), \\
\frac{\dot{R}}{R}=(-4.2 \pm 14.7) \times 10^{-11} \mathrm{~s} \mathrm{~s}^{-1}
\end{gathered}
$$

If we use the radius predicted by the evolutionary models for PG 1159-035 of Kawaler \& Bradley (1994), which is $R=0.025 \pm 0.005 R_{\odot}$, the radius change rate $\dot{R}$ would be

$$
\dot{R}=-0.073 \pm 0.256 \mathrm{~cm} \mathrm{~s}^{-1}
$$

or

$$
\dot{R}=-23 \pm 80 \mathrm{~km} \mathrm{yr}^{-1} \text {. }
$$

More realistic models must assume differential rotation, at least for the outer layer of the star. Detailed models of differentially rotating white dwarfs were constructed by Ostriker \& Bodenheimer (1968), and for all studied models, differential rotation is never very extreme, since $\Omega_{\text {equator }} / \Omega_{\text {center }}>0.2$. This means that the estimated value of $|\dot{R} / R|$ for a uniformly rotating star model must be considered as an upper limit. Thus, for PG 1159-035 (admitting a deviation of $3 \sigma$ )

$$
\left|\tau_{R}^{-1}\right|=\left|\frac{\dot{R}}{R}\right|<48 \times 10^{-11} \mathrm{~s} \mathrm{~s}^{-1},
$$

and hence

$$
|\dot{R}|<263 \mathrm{~km} \mathrm{yr}^{-1} .
$$

Note also that the estimate above assumes no frequency locking due to resonant coupling. Buchler, Goupil, \& Hansen (1997) show that frequency locking can occur for the most unstable mode or modes that predominantly sample the region of slower rotation in white dwarfs.

\section{SUMMARY AND CONCLUSIONS}

The secular variation of the $516 \mathrm{~s}$ period and its timescale was calculated by four methods described above, and the results are given in Table 4. All four methods give the same result within $1 \sigma$, but we emphasize that the most reliable method is the direct method, for the reasons mentioned in $\S 3$. The secular variation of the $516 \mathrm{~s}$ period is positive as predicted by the earlier theoretical models of Kawaler et al. (1985a) and Kawaler (1986) and by some models of Kawaler \& Bradley (1994). The magnitude, by contrast, is larger then predicted by any model calculated to date. This may be the first hint that cooling by neutrino emission is far more effective than current models describe, for these very hot, very dense stars.

The negative $\dot{P}$ value obtained by Winget et al. (1985) resulted from the classical $(O-C)$ diagram method, using underestimated values for the standard deviations of the phases, derived by least squares. This method masked the statistical significance of the result, aggravated by smallnumber statistics, even though Winget et al. were conservative with the treatment of the uncertainties, going so far as to multiply the uncertainty derived from least squares by a factor of 10. All later estimates (Winget \& Kepler 1988; Winget et al. 1991), though using larger data sets, used this first negative value as the initial value in their calculations, which explains the agreement between the results. These considerations also apply to the measurement of the secular variation of the $539 \mathrm{~s}$ period done by Costa et al. (1995), which used the same process, therefore perpetuating the cycle count miscalculation.

This erroneous result means that one should exercise extreme caution in estimating the precision of a measurement of phases of a sinusoidal signal in a multiperiodic data set. Estimates done from the least-squares process are, in general, much smaller than the actual values. More realistic values for the standard deviations must, if possible, be calculated using other techniques, such as the Monte Carlo simulations method. We also point out the advantage of using the direct method, at least for the case of pulsating stars whose periods vary rapidly enough, as is the case of

\begin{tabular}{|c|c|c|}
\hline Method & $\begin{array}{c}\dot{P} \\
\left(\times 10^{-11} \mathrm{~s} \mathrm{~s}^{-1}\right)\end{array}$ & $\begin{array}{c}\tau_{P} \\
\left(\times 10^{5} \mathrm{yr}\right)\end{array}$ \\
\hline Direct . & $+13.0 \pm 2.6$ & $1.26 \pm 0.25$ \\
\hline Maximum likelihood estimation...... & $+13.26 \pm 0.52$ & $1.23 \pm 0.05$ \\
\hline Modified $(O-C)$ algorithm & $+13.07 \pm 0.03$ & $1.25 \pm 0.05$ \\
\hline Nonlinear least squares.......... & $+13.298 \pm 0.003^{\mathrm{a}}$ & $1.2297 \pm 0.0002^{\mathrm{a}}$ \\
\hline
\end{tabular}
DOV stars. Cycle count errors have plagued most estimations of secular period changes.

The first attempt to model the observed PG 1159-035 pulsational spectrum with the KB models provides $\dot{P}=$ $+2.2 \times 10^{-11} \mathrm{~s} \mathrm{~s}^{-1}$ (Kawaler \& Bradley 1994). But, as the models are very sensitive to variation of the model parameters, some models yield values for $\dot{P}$ of order $10^{-10} \mathrm{~s} \mathrm{~s}^{-1}$.

TABLE 4

MEASUREMENTS OF THE $516 \mathrm{~s} \dot{P}^{\mathrm{a}}$

${ }^{a}$ Internal errors quoted to show the extreme overoptimism of the method. 
TABLE 5

$\dot{P}$ VAlues $\left(\times 10^{-11} \mathrm{~s} \mathrm{~s}^{-1}\right)$ FROM KB MODELS

\begin{tabular}{|c|c|c|c|c|c|}
\hline Parameters & $i b 593 b$ & $i b 593 i$ & $i b 593 x$ & $i b 593 y$ & $i b 603 h$ \\
\hline \multicolumn{6}{|c|}{$\dot{P}$ Values } \\
\hline$P=450 \mathrm{~s}(k=19) \ldots \ldots$ & 3.32 & 5.21 & 7.24 & 1.80 & 7.75 \\
\hline$P=493 \mathrm{~s}(k=21) \ldots \ldots$ & -3.15 & 6.90 & 0.29 & 0.72 & 7.48 \\
\hline$P=516 \mathrm{~s}(k=22) \ldots \ldots$ & -0.64 & 2.70 & 10.67 & -0.05 & 10.04 \\
\hline$P=539 \mathrm{~s}(k=23) \ldots \ldots$ & 2.91 & -0.81 & 11.52 & 1.39 & 7.78 \\
\hline \multicolumn{6}{|c|}{ Other Parameters } \\
\hline$M_{\mathrm{He}}\left(\times 10^{-3} M_{\star}\right) \ldots \ldots \ldots$ & 0.531 & 4.7 & 2.06 & 2.67 & 6.22 \\
\hline $\mathrm{C}: \mathrm{O}$ core $\ldots \ldots \ldots \ldots \ldots$ & $55: 45$ & $20: 80$ & $20: 80$ & $20: 80$ & $20: 80$ \\
\hline He:C:O surface .................... & $78: 21: 1$ & $78: 21: 1$ & $58: 27: 15$ & $58: 27: 15$ & $78: 21: 1$ \\
\hline$M / M_{\odot} \ldots \ldots \ldots \ldots \ldots \ldots$ & 0.59 & 0.59 & 0.59 & 0.59 & 0.60 \\
\hline
\end{tabular}

Table 5 shows the $\dot{P}$ values derived from KB models with $T_{\text {eff }} \simeq 140,000 \mathrm{~K}$ (P. Bradley 1998, private communication). The sequences $i b 593 x$ and $i b 603 h$ present larger $\dot{P}$ values, close to our value, indicating that high values for $\dot{P}$ from KB models are possible. The models with large mode trapping show that $\dot{P}$ values are oscillatory with overtone number $k$. Models with an essentially monotonic sequence of $\dot{P}$ values indicate that mode-trapping effects on $\dot{P}$ are swamped by cooling. Therefore, it is important to measure the rates of change of other pulsational periods of $\mathrm{PG}$ $1159-035$ to study mode trapping. We are working on the estimates of the secular variation of the largest possible number of periods present on PG $1159-035$ pulsational spectra.

The analysis of the rotational splitting of the $517 \mathrm{~s}$ mode, though not statistically significant, provides some interesting results. The central component of the triplet shows an apparent variation from 1989 to 1993 of $\dot{P}_{517}=(+23 \pm 23)$ $\times 10^{-11} \mathrm{~s} \mathrm{~s}^{-1}$, consistent with the expectation that the prograde component $(m=+1)$ of the triplet has a secular variation smaller than that of the central component $(m=0)$, because the star is contracting. From this estimate, we can constrain the rate of change of the rotational period: $\dot{P}_{\text {rot }}=(-1.0 \pm 3.5) \times 10^{-5} \mathrm{~s} \mathrm{~s}^{-1}$, or less than a few minutes per year.

Brickhill, A. J. 1975, MNRAS, 170, 404

Buchler, J. R., Goupil, M.-J., \& Hansen, C. J. 1997, A\&A, 321, 159

Costa, J. E. S. 1996, M.Sc. thesis, Inst. Fís. Univ. Federal do Rio Grande do Sul, Brazil

Costa, J. E. S., Kepler, S. O., \& Winget, D. E. 1995, Baltic Astron., 4, 334

Deeming, T. J. 1975, ApJ, 36, 137

de Jager O. C., Meintjes, P. J., \& O'Donoghue, D. 1994, MNRAS, 267, 577

Dreizler, S. 1998, Baltic Astron., 7, 71

Green, R. F. 1977, Ph.D. thesis, California Inst. Technol.

Hansen, C. J., Cox, J.P., \& Van Horn, H. M. 1977, ApJ, 217, 151

Kawaler, S. D. 1986, Ph.D. thesis, Univ. Texas, Austin

Kawaler, S. D., \& Bradley, P. A. 1994, ApJ, 427, 415

Kawaler, S. D., Hansen, C. J., \& Winget, D. E. 1985a, ApJ, 295, 547

Kawaler, S. D., Winget, D. E., \& Hansen, C. J. 1985b, ApJ, 298, 752

Kepler, S. O., Robinson, E. L., Nather, R. E., \& McGraw, J. T. 1982, ApJS, 254, 676

Kepler, S. O., Winget, D. E., Nather, R. E., Bradley, P. A., Grauer, A. D., Fontaine, G., Bergeron, P., Vauclair, G., Claver, C. F., Marar, T. M. K., Seetha, S., Ashoka, B. N., Mazeh, T., Leibowitz, E., Dolez, N., Chevreton, M., Barstow, M. A., Clemens, J. C., Kleinman, S. J., Sansom, A. E., Tweedy, R. W., Kanaan, A., Hine, B. P., Provencal, J. L., Wesemael, F., Wood, M. A., Brassard, P., Solheim, J. -E., \& Emanuelsen, P.-I. 1991, ApJ, 378, L45

McGraw, J. T., Starrfield, S. G., Liebert, J., \& Green, R. F. 1979, in IAU Colloq. 53, White Dwarfs and Variable Degenerate Stars, ed. H. M. Van Horn \& V. Weidemann (Rochester: Univ. Rochester), 377
Although the rate of rotation period change is only a rough estimate, it demonstrates the potential of this technique. The importance of the new technique arises because rotation changes are sensitive to radius change and insensitive to temperature change. The examination of these changes separately offers the exciting possibility of understanding this rapid but mysterious evolutionary phase in detail. Rotational splitting period change analysis allows us to derive radius change timescales, important parameters in stellar evolutionary theory. For statistically significant results, new observations of PG 1159-035 with WET will be necessary. This will also allow us to infer the rotational law and thereby to improve the determination of evolutionary timescales. Furthermore, new theoretical models for PG $1159-035$ using the $\dot{P}_{516}$ estimates must be calculated.

We deeply appreciate Paul Bradley's very useful suggestions, which improved the presentation of the paper. Most of the calculations were made using the Cray YMP-2E computer at the Centro Nacional de Supercomputação da Universidade Federal do Rio Grande do Sul.

\section{REFERENCES}

Nather, R. E., Winget, D. E., Clemens, J. C., Hansen, C. J., \& Hine, B. P. 1990, ApJ, 361, 309

O'Donoghue, D. 1994, MNRAS, 270, 222

Ostriker, J. P., \& Bodenheimer, P. 1968, ApJ, 151, 1089

Press, W. H., Teukolsky, S. A., Vetterling, W. T., \& Flannery, B. P. 1986, Numerical Recipes in FORTRAN (2d ed.; Cambridge: Cambridge Univ. Press), 209

Schwarzenberg-Czerny, A. 1989, MNRAS, 241, 153

Sion, E. M., Greenstein, J. L., Landstreet, J. D., Liebert, J., Shipman, H. L., \& Wegner, G. 1983, ApJ, 269, 253

Stellingwerf, R. F. 1978, ApJ, 224, 953

Werner, K. 1995, Baltic Astron., 4, 340

Winget, D. E., Hansen, C. J., \& Van Horn, H. M. 1983, Nature, 303, 781

Winget, D. E., \& Kepler, S. O. 1988, in Pulsations in Classical and Cataclysmic Variable Stars, ed. J. P. Cox \& C. J. Hansen (Boulder: Univ. Colorado Press), 46

Winget, D. E., Kepler, S. O., Robinson, E. L., Nather, R. E., \& O’Donoghue, D. 1985, ApJ, 292, 606

Winget, D. E., Nather, R. E., Clemens, J. C., Provencal, J. L., Kleinman, S. J., Bradley, P. A., Wood, M. A., Claver, C. F., Frueh, M. L., Grauer, A. D., Hine, B. P., Hansen, C. J., Fontaine, G., Achilleos, N., Wickramasinghe, D. T., Marar, T. M. K., Seetha, S., Ashoka, B. N., O'Donoghue, D., Warner, B., Kurtz, D. W., Buckley, D. A., Brickhill, J., Vauclair, G., Dolez, N., Chevreton,M., Barstow, M. A., Solheim, J. E., Kanaan, A., Kepler, S. O., Henry, G. W., \& Kawaler, S. D. 1991, ApJ, 378,326 